\title{
Die Ausgestaltung der Umweltrechtsbehelfe für Verbände seit der UmwRG-Novelle 2017*
}

\author{
Annette Guckelberger
}

\begin{abstract}
(C) Der/die Autor(en) 2020
Angesichts der Ausweitung der umweltrechtsbehelfsfähigen Gegenstände sowie des partiellen Wegfalls der materiellen Präklusion enthält die UmwRG-Novelle 2017 eine Reihe von Vorschriften zur Eindämmung der daraus resultierenden Effekte. Deren Bedeutungsgehalt bedarf noch weiterer Konkretisierung in der Praxis. Ferner stehen die Anerkennungsvoraussetzungen für Umweltorganisationen nicht nur im Fokus des Bundesgesetzgebers, sondern auch des Aarhus Compliance Committee (ACC).
\end{abstract}

\section{Einführung}

Wie im Beitrag „Die Erweiterung der umweltrechtsbehelfsfähigen Gegenstände auf dem Prüfstand " aufgezeigt wurde, hat der Gesetzgeber zur Erfüllung seiner Verpflichtungen aus Art. 9 Abs. $3 \mathrm{AK}^{2}$ sowie aus dem Unionsrecht durch die UmwRG-Novelle 2017 den Kreis der umweltrechtsbehelfsfähigen Gegenstände erweitert, aber wohl nicht weit genug. Zugleich wurden weitere Regelungen getroffen. Im Zuge der teilweisen Zurückführung der materiellen Präklusion wurde eine Vorschrift über missbräuchliches oder unredliches Verhalten eingeführt, welche die innerprozessuale Präklusion strenger ausgestaltet, und es wurden die Heilungsvorschriften erweitert. Ausgehend von der Rechtsprechung $\mathrm{zu}$ diesen Regelungen wird eine Bestandsaufnahme zu weiteren Neuerungen der UmwRG-Novelle 2017 vorgenommen, die auch Kritik und Änderungsvorschläge einbezieht.

\section{Rechtsbehelfsbefugnis der Vereinigungen gemäß $₫ 2$ Abs. 1 UmwRG}

Da Art. 9 Abs. 2 AK anders als dessen Absatz 3 keine Rechtsgrundlage für eine Beschränkung der Umweltrechtsbehelfe von Verbänden auf mögliche Rechtsverletzungen mit einem Umweltbezug enthält, wurden zur Umsetzung der Entscheidung V/9h der 5. Vertragsstaatenkonferenz zur $\mathrm{AK}^{3}$ in $\$ 2$ Abs. 1 Nr. 1 UmwRG die Worte ,die dem Umweltschutz dienen" gestrichen. ${ }^{4}$ Nunmehr können nach $\$ 3$ UmwRG anerkannte Vereinigungen ohne das Erfordernis einer möglichen Verletzung in eigenen Rechten Rechtsbehelfe gegen Entscheidungen nach $\$ 1$ Abs. 1 S. 1 UmwRG oder deren Unterlassen einlegen, wenn folgende Voraussetzungen erfüllt sind: Erstens muss die Vereinigung geltend machen, dass eine solche Entscheidung oder Unterlassung Rechtsvorschriften, die für die Entscheidung von Bedeutung sein können, widerspricht. Des Weiteren muss sie darlegen, durch die Entscheidung oder Unterlassung in ihrem satzungsgemäßen Aufgabenbereich der Förderung der Ziele des Umweltschutzes berührt zu sein. Da der EuGH die Anordnung einer materiellen Präklusion bei Art. 9 Abs. 2 AK für unzulässig gehalten hat, wird nunmehr bei Rechtsbehelfen i.S.d. $\$ 1$ Abs. 1 S. 1 Nrn. 1-2b

Prof. Dr. Annette Guckelberger,

Lehrstuhl für Öffentliches Recht,

Universität des Saarlandes,

Saarbrücken, Deutschland
UmwRG nur noch verlangt, dass die Vereinigung im Falle eines Verfahrens zur Beteiligung berechtigt war (\$2 Abs. 1 Nr. 3 lit. a) UmwRG). ${ }^{5}$ Es kommt nicht mehr darauf an, ob sie sich in der Sache gemäß den geltenden Rechtsvorschriften geäußert hat oder ihr entgegen diesen Rechtsvorschriften keine Gelegenheit zur Äußerung gegeben wurde. Aufgrund der anderen Fassung des Art. 9 Abs. 3 AK hielt man jedoch bei Entscheidungen nach $\$ 1$ Abs. $1 \mathrm{~S} .1 \mathrm{Nr} .4$ UmwRG an einer materiellen Präklusion fest. Abhängig von der Art des Bebauungsplans ist bei SUP-pflichtigen Bebauungsplänen als Entscheidungen i.S.d. \$1 Abs. 1 S. 1 Nr. 4 UmwRG der Frage nach der ordnungsgemäßen ÄuBerung im Verwaltungsverfahren nachzugehen, bei UVPpflichtigen Bebauungsplänen als Entscheidungen nach $₫ 1$ Abs. 1 S. 1 Nr. 1 UmwRG hingegen nicht. ${ }^{6}$

Das Beteiligungserfordernis des $\$ 2$ Abs. 1 S. 1 Nr. 3 UmwRG besteht nur bei Entscheidungen nach $\$ 1$ Abs. 1 S. 1 Nrn. 1-2b und Nr. 4 UmwRG. Es erlangt bei einer $\$ 1$ Abs. 1 S. 1 Nr. 5 UmwRG unterfallenden immissionsschutzrechtlichen Genehmigung im vereinfachten Verfahren keine Relevanz. ${ }^{7} \mathrm{Ob}$ eine Beteiligungsberechtigung besteht, richtet sich nach dem einschlägigen Fachrecht, etwa nach $\$ 3$ BauGB, $\$ 10$ Abs. 3 BImSchG, \18 UVPG, $\$ 70$ Abs. 1 WHG i. V.m. $\$ 73$ VwVfG oder folgt ggf. zur Herstellung von Unionsrechtskonformität aus dem Unionsrecht. ${ }^{8}$

\subsection{Teilweises Erfordernis der Rüge einer Verletzung umweltbezogener Vorschriften}

Während die Vereinigungen bei Entscheidungen nach $\$ 1$ Abs. 1 S. 1 Nr. 1 UmwRG grundsätzlich jeglichen Rechtsverstoß rügen können, ${ }^{9}$ wird ihre Rügebefugnis bei Rechts-

^) Es handelt sich um den dritten von fünf Beiträgen, welche einer Langfassung ihres Vortrag in Berlin am 2.12.2019 auf dem „Forum Umweltrechtsschutz 2019: Erfahrungen mit der Novelle des Umwelt-Rechtsbehelfsgesetzes (UmwRG) seit 2017“, veranstaltet durch das Unabhängige Institut für Umweltfragen, entsprechen.

1) NuR 2020, 217

2) Übereinkommen über den Zugang zu Informationen, die Öffentlichkeitsbeteiligung an Entscheidungsverfahren und den Zugang $\mathrm{zu}$ Gerichten in Umweltangelegenheiten v. 25.6.1998.

3) BT-Drs. 18/9526, S. 44; s. auch Schlacke, NVwZ 2019, 1392, 1393.

4) BT-Drs. $18 / 9526$, S. 38

5) BT-Drs. 18/9526, S. 38; s. auch Schieferdecker, in: Hoppe/Beckmann/Kment, UVPG/UmwRG, 5. Aufl. 2018, \2 UmwRG Rdnr. 23.

6) OVG Lüneburg, Urt. v. 15.11.2018 - 1 KN 29/17, NVwZ-RR 2019, 631, 632.

7) VG Hannover, Beschl. v. 28.3.2019 - 4 B 5526/18, Rdnr. 84, juris; zur anderen Rechtslage bei immissionsschutzrechtlichen Genehmigungen mit Beteiligung VG Hannover, Beschl. v. 28. 2. $2019-$ 12 B 6923/18, Rdnr. 72, juris.

8) So VG Sigmaringen, Urt. v. 14.11.2018-10 K 118/17, Rdnr. $163 \mathrm{ff}$., juris; dazu auch Schlacke, NVwZ 2019, 1392, 1400.

9) BT-Drs. 18/9526, S. 38, aber darauf verweisend, dass sich eine Beschränkung aus dem satzungsgemäßen Aufgabenbereich der Vereinigung ergibt. 
behelfen gegen eine Entscheidung nach $\$ 1$ Abs. 1 S. 1 Nrn. 2a-6 UmwRG oder deren Unterlassen beschränkt. Gemäß $\$ 2$ Abs. 1 S. 2 UmwRG muss bei diesen eine Verletzung umweltbezogener Vorschriften geltend gemacht werden. Soweit gewisse Vorhaben, die eigentlich \$1 Abs. 1 S. 1 Nr. 5 UmwRG unterfallen, zugleich die Kriterien des Art. 6 Abs. 1 lit. b) AK erfüllen und damit die Rechtsschutzvorgabe des Art. 9 Abs. 2 AK einschlägig ist, ist bei diesen aus Gründen des Unions- sowie Völkerrechts von der Geltendmachung einer Verletzung umweltbezogener Vorschriften abzusehen. ${ }^{10}$ Da der Begriff der umweltbezogenen Vorschriften auch bei $\$ 1$ Abs. 1 S. 1 Nrn. 5, 6 UmwRG gebraucht wird, ist insoweit auf die Legaldefinition in $\$ 1$ Abs. 4 UmwRG zu rekurrieren. Bei den umweltbezogenen Rechtsvorschriften i.S.d. UmwRG handelt es sich um Bestimmungen, die sich zum Schutz von Mensch und Umwelt auf (1.) den Zustand von Umweltbestandteilen i.S.v. $\$ 2$ Abs. 3 Nr. 1 UIG oder (2.) Faktoren i.S.v. \$2 Abs. 3 Nr. 2 UIG beziehen. In den Worten der Materialien wird so eine 1:1-Umsetzung der EU-Umweltinformationsrichtlinie sowie der dahinterstehenden Begriffe der AK vorgenommen, ferner wird auf die eine weite Auslegung vornehmende Spruchpraxis des ACC verwiesen. ${ }^{11}$ Es reicht aus, wenn die infrage stehenden Vorschriften auch dem Schutz der Umwelt dienen. ${ }^{12}$

Nicht nur Rechtsvorschriften, in deren Überschrift oder Text explizit das Wort „Umwelt“ gebraucht wird, sind umweltbezogene Rechtsvorschriften. ${ }^{13}$ Ausschlaggebend nach der Legaldefinition ist vielmehr, ob die jeweilige Rechtsvorschrift in irgendeiner Weise einen Bezug zu den Umweltbestandteilen oder Faktoren i.S.d. \$2 Abs. 3 UIG aufweist. ${ }^{14}$ Deshalb können auch Vorschriften aus dem BauGB umweltbezogene Rechtsvorschriften sein, sofern sie sich konkret auf die Umwelt beziehen, ${ }^{15}$ wie dies bei \35 Abs. 3 S. 1 Nrn. 3, 5 BauGB,${ }^{16}$ nicht jedoch den baurechtlichen Vorschriften zum Erschließungsbeitrag, zu Sanierungssatzungen oder zur Städtebauförderung der Fall ist. ${ }^{17}$ Das planungs- oder baurechtliche Abwägungsgebot stellt bei Einfließen von Umweltbelangen in die Abwägung eine umweltbezogene Rechtsvorschrift dar. ${ }^{18}$ Ein weiteres Beispiel für eine umweltbezogene Rechtsvorschrift bildet der Vorsorgegrundsatz des $₫ 5$ Abs. 1 Nr. 2 BImSchG. ${ }^{19}$ Umweltbezogen sind nicht nur Vorschriften, die materiell-rechtliche Vorgaben zum Schutz bestimmter Umweltbestandteile, etwa in Gestalt der Festlegung von Grenzwerten, oder in Bezug auf Umweltfaktoren enthalten, sondern auch verfahrensrechtliche Vorschriften, welche die Notwendigkeit einer eingehenden behördlichen Prüfung davon abhängig machen, ob und in welchem Ausmaß nachteilige Auswirkungen auf bestimmte Umweltschutzgüter verursacht werden können. ${ }^{20}$ Das OVG Lüneburg sah in $₫ 37$ Abs. 1 VwVfG und $₫ 23$ Abs. 2 Nr. 3 der 9. BImschV umweltbezogene Bestimmungen, sofern sie zur Effektuierung des materiellen Umweltrechts beitragen. ${ }^{21}$

\section{2 Änderungsvorschlag in einem Gutachten für den Normenkontrollrat}

Nach dem Gutachten für den Normenkontrollrat zur Beschleunigung verwaltungsgerichtlicher Vorhaben lässt sich aus $\$ 2$ Abs. 1 S. 2 UmwRG nicht hinreichend eindeutig entnehmen, ob für die Geltendmachung einer Verletzung umweltbezogener Vorschriften bei Plänen und Programmen i.S.d. $\$ 1$ Abs. 1 S. 1 Nr. 4 UmwRG der Vortrag der Einstellung und Abarbeitung von Umweltbelangen in der Abwägung genügt oder ob tatsächlich Umstände vorgetragen werden müssen, aus denen sich hinsichtlich der Umweltbelange die Möglichkeit eines Abwägungsfehlers ergibt. ${ }^{22} \mathrm{Da}$ das BVerwG seit langem von Individualklägern in Normenkontrollverfahren einen solchen Tatsachenvortrag verlange, spreche viel dafür, dass in denjenigen von Umweltvereinigungen nichts anderes gelten könne. Da es kaum Bebauungspläne ohne eine Abwägung auch umweltrelevanter Belange gibt, wäre andernfalls in den Normenkontrollverfahren die Antragsbefugnis der Umweltverbände fast immer zu bejahen. ${ }^{23}$ Deshalb soll $\$ 2$ Abs. 1 UmwRG ein Satz 3 mit folgendem Inhalt hinzugefügt werden: ,Soweit im Falle von Entscheidungen nach $\$ 1$ Abs. 1 Satz 1 Nr. 4 eine fehlerhafte Behandlung von Umweltbelangen in einer Abwägung geltend gemacht wird, sind die hierfür maßgeblichen Umstände zu benennen."24 Auf diese Weise würde eine prozessual ohnehin bestehende Obliegenheit der Verbände ohne Veränderung der materiellen Maßstäbe klargestellt. Damit ginge ein Beschleunigungseffekt einher, da die Verbände beizeiten substantiiert vortragen müssten, was dem Gericht und den Passivbeteiligten eine frühzeitige Befassung mit der Angelegenheit ermögliche sowie unsubstantiierte Rechtsbehelfe umgehend $a b$ weisungsreif mache..$^{25}$

10) Fischer-Hüftle, NuR 2018, 735, 740.

11) BT-Drs. 18/9526, S. 36.

12) Dikaios, Überindividueller Umweltrechtsschutz am Beispiel der altruistischen Verbandsklage in der deutschen, griechischen und europäischen Rechtsordnung, 2018, S. 350. Dagegen für eine einschränkende Auslegung, wenn Umweltbelange z.B. in einer Ermessensentscheidung erkennbar keine Bedeutung entfalten können, Schieferdecker, in: Hoppe/Beckmann/Kment, UVPG/ UmwRG, 5. Aufl. 2018, \$1 UmwRG Rdnr. 112.

13) VGH München, Beschl. v. 11.4.2018 - 2 CS 18198, NuR 2019, 483; s. auch Fellenberg/Schiller, in: Landmann/Rohmer, Umweltrecht, Stand: 6/2019, \$1 UmwRG Rdnr. 161.

14) VGH München, Beschl. v. 11.4.2018 - 2 CS 18198, NuR 2019, 483; VG Neustadt a.d. W., Beschl. v. 26.9.2019 - 5 L 963/19. NW, Rdnr. 19, juris.

15) VG Köln, Beschl. v. 18. 4.2019 - 2 L 557/19, Rdnr. 26, juris; VG Hannover, Beschl. v. 28.3.2019 - 4 B 5526/18, Rdnr. 71, juris.

16) OVG Lüneburg, Beschl. v. 4.9.2018 - 1 ME 65/18, NuR 2018 , 777, 779; VGH München, Beschl. v. 5.4.2019 - 22 CS 18.2572, 22 CS 19.23, Rdnr. 42, juris. Der VGH München, Beschl. v. 11.4.2018 - 2 CS 18198, NuR 2019, 483, hat dagegen bei $\$ 30$ BauGB eine umweltbezogene Vorschrift verneint.

17) VGH München, Beschl. v. 11.4.2018 - 2 CS 18198, NuR 2019, 483

18) VGH Mannheim, Urt. v. 20.11.2018 - 5 S 2138/16, Rdnr. 305, juris; Fellenberg/Schiller, in: Landmann/Rohmer, Umweltrecht, Stand: 6/2019, \&1 UmwRG Rdnr. 163.

19) VG Hannover, Beschl. v. 28.3.2019 - 4 B 5526/18, Rdnr. 145, juris.

20) VGH München, Beschl. v. 5.4.2019 - 22 CS 18.2572, 22 CS 19.23, Rdnr. 63, juris; s. auch Berkemann, DVB1. 2020, 1, 4 f.; Schieferdecker, in: Hoppe/Beckmann/Kment, UVPG/UmwRG, 5. Aufl. 2018, \$1 UmwRG Rdnr. 111.

21) OVG Lüneburg, Urt. v. 25.10.2018 - 12 LB 118/16, Rdnr. 156, juris.

22) Ewer, Möglichkeiten zur Beschleunigung verwaltungsgerichtlicher Verfahren über Vorhaben zur Errichtung von Infrastruktureinrichtungen und Industrieanlagen v. 1.4.2019, Stand 11.11.2019, abrufbar unter https://www.normenkontrollrat.bund.de/resource/ blob/72494/1600406/f0613bfaa6ea13b6a35d756672387d29/ 2019-04-17-nkr-gutachten-2018-data.pdf?download=1, S. 96.

23) Ewer, Möglichkeiten zur Beschleunigung verwaltungsgerichtlicher Verfahren über Vorhaben zur Errichtung von Infrastruktureinrichtungen und Industrieanlagen v. 1.4.2019, Stand 11.11.2019, abrufbar unter https://www.normenkontrollrat.bund.de/resource/ blob/72494/1600406/f0613bfaa6ea13b6a35d756672387d29/ 2019-04-17-nkr-gutachten-2018-data.pdf?download=1, S. 97.

24) Ewer, Möglichkeiten zur Beschleunigung verwaltungsgerichtlicher Verfahren über Vorhaben zur Errichtung von Infrastruktureinrichtungen und Industrieanlagen v. 1.4.2019, Stand 11.11.2019, abrufbar unter https://www.normenkontrollrat.bund.de/resource/ blob/72494/1600406/f0613bfaa6ea13b6a35d756672387d29/ 2019-04-17-nkr-gutachten-2018-data.pdf?download=1, S. 98.

25) Ewer, Möglichkeiten zur Beschleunigung verwaltungsgerichtlicher Verfahren über Vorhaben zur Errichtung von Infrastruktureinrichtungen und Industrieanlagen v. 1.4.2019, Stand 11.11.2019, abrufbar unter https://www.normenkontrollrat.bund.de/resource/ blob/72494/1600406/f0613bfaa6ea13b6a35d756672387d29/ 2019-04-17-nkr-gutachten-2018-data.pdf?download=1, S. 98. 


\subsection{Unterschiedliche Positionen in Bezug auf Denkmal- und Tierschutzvorschriften}

Zugegebenermaßen kann die Abgrenzung zwischen umweltbezogenen- und nicht umweltbezogenen Vorschriften Schwierigkeiten bereiten. Umstritten ist z.B., wie mit denkmal- oder tierschutzrechtlichen Bestimmungen umzugehen ist. Fellenberg/Schiller gehen davon aus, dass der Gesetzgeber auf die Erwähnung weiterer Schutzgüter, wie etwa das Klima, aber auch Kulturstätten und Bauwerke nach $\$ 2$ Abs. 3 Nr. 6 UIG, verzichtet hat. ${ }^{26}$ Angesichts der weiten Auslegung des Begriffs der umweltbezogenen Bestimmungen durch das ACC und der Verwendung eines anderen Umweltbegriffs etwa in Art. 3 lit. a) Var. 2 UVPRichtlinie, der sich auf die Fauna, also die Gesamtheit aller in einem natürlichen Gebiet lebenden Tiere (s. auch die Erwähnung der natürlichen Lebensgrundlagen sowie des Artenschutzes in $\$ 2$ Abs. $3 \mathrm{Nr} .1 \mathrm{UIG}$ ), sowie nach Art. 3 lit. c) Var. 2 UVP-Richtlinie auf das kulturelle Erbe bezieht, ist jedoch von einer solchen restriktiven Handhabung dieser Vorschrift abzuraten. Verletzungen von Art. 9 Abs. 3 AK sowie des Unionsrechts lassen sich vermeiden, indem man die Beschreibungen des Zustands von Umweltbestandteilen sowie der Faktoren in $\$ 2$ Abs. 3 Nrn. 1, 2 UIG aufgrund des dort verwendeten Worts „wie“ nicht als abschließend versteht. ${ }^{27}$ Was den Tierschutz anbetrifft, wird vermutlich erst eine Involvierung des EuGH für die notwendige Klarheit sorgen. Das BVerwG entschied jüngst, dass sich unter den Begriff der Umwelt i. S. d. UIG nur Tiere als Teile der natürlichen Lebensräume und die Artenvielfalt an sich, nicht aber tierschutzrechtliche Belange fassen lassen. ${ }^{28} \mathrm{Im}$ Unterschied zum Umweltinformationsrecht wird in Art. 3 lit. a) UVP-Richtlinie die Formulierung „Mensch, Fauna und Flora" verwendet. Teilweise wird diese Formulierung sehr weit als Erfassung der Tier- und Pflanzenwelt im umfassenden Sinn verstanden, also unter Einschluss u.a. von Haustieren, ${ }^{29}$ so dass man darunter möglicherweise auch Nutz- und Schlachttiere fassen könnte. ${ }^{30}$ Blickt man auf die Umschreibung der „Informationen über die Umwelt" in Art. 2 Nr. 3 lit. a) AK, spricht die dortige Erwähnung von Wasser, Boden, Land, Landschaft und natürlichen Lebensräumen, die Artenvielfalt und ihre Bestandteile sowie die Nennung der Brutstätten seltener Tierarten in Art. 4 Abs. 4 lit. h) AK für eine engere Lesart. Die im Anhang I zu Art. 6 Abs. 1 lit. a) AK u.a. erwähnten Anlagen zum Schlachten mit einer Schlachtkapazität von mehr als 50 Tonnen pro Tag oder Anlagen zur Beseitigung oder Verwertung von Tierkörpern und tierischen Abfällen mit einer Verarbeitungskapazität von mehr als 10 Tonnen pro Tag verfügen über einen Anlagenbezug. So erklärt sich auch, warum mehrere Bundesländer zwischenzeitlich eine eigenständige tierschutzrechtliche Verbandsklage eingeführt haben. ${ }^{31}$

\subsection{Bezug zum satzungsmäßigen Aufgabenbereich der Vereinigung}

Der Vollständigkeit halber sei darauf hingewiesen, dass in letzter Zeit einige Gerichtsentscheidungen zu dem schon längere Zeit bestehenden Erfordernis des $\$ 2$ Abs. $1 \mathrm{~S}$. 1 Nr. 2 UmwRG ergangen sind, nämlich des Geltendmachens der Umweltschutzvereinigung, durch die jeweilige Entscheidung oder deren Unterlassen in ihrem satzungsmäBigen Aufgabenbereich berührt zu sein. Mit diesem Erfordernis geht eine Beschränkung der Rügebefugnis einher, weil außerhalb des Satzungszwecks liegende Rechtsverstöße für die Umweltorganisation gerichtlich nicht durchsetzbar sind..$^{32}$ Zutreffend stellen sich die Gerichte auf den Standpunkt, dass die Novellierung des $₫ 2$ Abs. 1 S. 1 Nr. 1 UmwRG nicht durch einen zu eng gefassten Satzungsbezug ausgehebelt werden darf. ${ }^{33}$ Deshalb genügt es, wenn sich eine mittelbare Verknüpfung der Satzungsziele der Vereinigung mit dem geltend gemachten Rechtsverstoß und seinen Folgen herstellen lässt. ${ }^{34}$ Infolge einer weiten Auslegung des Satzungszwecks kann daher eine auf den $\mathrm{Na}$ turschutz ausgerichtete Vereinigung Verletzungen immissionsschutzrechtlicher Vorschriften rügen, soweit durch die angegriffene Entscheidung die Realisierung des Schutzguts Natur beeinträchtigt wird. ${ }^{35} \mathrm{Da}$ nach dem Gesetzeswortlaut das „Berührtsein“ im satzungsgemäßen Aufgabenbereich genügt, kann es bei der Zulässigkeitsprüfung nicht darauf ankommen, ob sich diesem tatsächlich jede einzelne Rüge zuordnen lässt. ${ }^{36}$ Der von den Verwaltungsgerichten vorgenommenen weiten Auslegung ist zuzustimmen. ${ }^{37}$ In seinem Bericht vom 31.7.2017 betonte das ACC, dass den Nichtregierungsorganisationen der Zugang zu Gericht nicht faktisch verwehrt und daher nur eine allgemeine $\mathrm{Be}$ ziehung zwischen den satzungsmäßigen Zielen und den Gründen des Rechtsbehelfs verlangt werden darf. ${ }^{38}$ Gemäß der Entscheidung des EuGH in der Rechtssache Djurgarden kann der nationale Gesetzgeber von der gegen ein Projekt vorgehenden Vereinigung lediglich verlangen, dass sie „ein natur- und umweltschutzbezogenes Ziel hat". ${ }^{39}$

\section{Anerkennungsvoraussetzungen}

Anlässlich der UmwRG-Novelle wurde in $\$ 3$ Abs. 1 S. 3 UmwRG klarstellend aufgenommen, dass in der Anerkennung der räumliche Bereich anzugeben ist, auf den sich diese bezieht. Bereits nach bestehendem Recht würde die Anerkennung von Vereinigungen durch Landesbehörden lediglich für bestimmte Regionen erfolgen, wenn die Vereinigung nach ihrer Satzung und tatsächlichen Tätigkeit nur in dieser Region tätig wird. ${ }^{40}$ Infolgedessen bestehe deren Rechtsbehelfsbefugnis nach $₫ 2$ Abs. 1 Nr. 2 UmwRG

26) Fellenberg/Schiller, in: Landmann/Rohmer, Umweltrecht, Stand: 6/2019, \$1 UmwRG Rdnr. 161.

27) So auch Bunge, in: ders., UmwRG, 2. Aufl. 2019, \&1 Rdnr. 204 Zum Denkmalschutz Mast, ZRP 2019, 237, 238; s. auch KleineTebbe, in: Martin/Krautzberger, Handbuch Denkmalschutz und Denkmalpflege, 4. Aufl. 2017, Teil A Rdnr. 39 ff. Für diese Auslegung spricht auch, dass das BVerwG, NVwZ 2013, 642, 643, vor der UmwRG-Novelle den Begriff des „Umweltschutzes“ anhand des UVPG bestimmte.

28) BVerwG, Urt. v. 30.1.2020 - 10 C 11.19, NVwZ 2020, 880 m. Anm. Gerhold.

29) So Schomerus, in: ders./Schrader/Wegener, UIG, 2. Aufl. 2002, $\$ 3$ Rdnr. 11

30) Eine solche weite Auslegung in Erwägung ziehend OVG Lüneburg, Urt. v. 27.2.2018 - 2 LC 58/17, Rdnr. 36, juris.

31) Baden-Württemberg, Bremen, Hamburg, Niedersachsen, Nordrhein-Westfalen (inzwischen nicht mehr), Rheinland-Pfalz, Saarland, Schleswig-Holstein. S. dazu etwa Groß, Die Rechtsdurchsetzung von Tierbelangen insbesondere durch tierschutzrechtliche Verbandsklagen, 2018; Ley, Das Instrument der Tierschutz-Verbandsklage, 2018; Kloepfer/Kluge (Hrsg.), Die tierschutzrechtliche Verbandsklage, 2016.

32) VG Regensburg, Beschl. v. 21.11.2018 - RN 7 S 18.1756 , Rdnr. 62, juris; Kment, NVwZ 2018, 921, 923.

33) BVerwG, Urt. v. 11.10.2017 - 9 A 14.16, BVerwGE 160, 78, 81 Rdnr. 10; VG Regensburg, Beschl. v. 21.11.2018 - RN 7 S 18.1756, Rdnr. 62, juris; Berkemann, DVB1. 2020, 1, 3.

34) BVerwG, Urt. v. 11.10.2017 - 9 A 14.16, BVerwGE 160, 78 , 81 Rdnr. 10; VG Regensburg, Beschl. v. 21.11.2018 - RN 7 S 18.1756, Rdnr. 62, juris; Fellenberg/Schiller, in: Landmann/ Rohmer, Umweltrecht, Stand: 6/2019, \$2 UmwRG Rdnr. 20.

35) VG Regensburg, Beschl. v. 21.11.2018 - RN 7 S 18.1756, Rdnr. 62; Kment, NVwZ 2018, 921, 923.

36) OVG Hamburg, Urt. v. 15.8.2018 - 1 Es 1/18.P, ZUR 2019, 37, 39 (wobei etwas anderes für die Begründetheitsprüfung gelten mag).

37) Für eine weite Auslegung auch Schieferdecker, in: Hoppe/Beckmann/Kment, UVPG/UmwRG, 5. Aufl. 2018, \$2 UmwRG Rdnr. 19

38) ACC, ZUR 2018, 23, 24

39) EuGH, Urt. v. 15.10.2009 - C-263/08, ECLI:EU:C:2009:63, Rdnr. 46.

40) BT-Drs. 18/9526, S. 39 
,nur in Bezug auf Vorhaben, die in dieser konkreten Region verwirklicht werden sollen oder auf diese Region Auswirkungen haben können". ${ }^{41}$

\subsection{Gewähr für eine sachgerechte Aufgabenerfüllung}

Last but not least wurde durch die UmwRG-Novelle 2017 in $\$ 3$ Abs. 1 S. 1 Nr. 3 UmwRG verdeutlicht, dass zur Gewähr einer sachgerechten Aufgabenerfüllung durch die Vereinigung insbesondere eine sachgerechte Beteiligung an behördlichen Entscheidungsverfahren gehört. Dazu ist nach der Rechtsprechung eine Prognose ausgehend von der bisherigen Verbandstätigkeit vorzunehmen, bei der auch zu berücksichtigen ist, ob die Vereinigung ein Mindestmaß an Kooperationsbereitschaft gegenüber den Behörden erkennen lässt. ${ }^{42}$ Daran fehlt es jedoch nicht bereits deshalb, weil die Vereinigung als hartleibiger oder schwieriger Verhandlungspartner agiert. ${ }^{43}$ Die Anerkennung darf nach dem VG Wiesbaden erst dann versagt werden, wenn die Vereinigung „,den Naturschutz kompromisslos und mit Mitteln verficht, die der Sache nach unangemessen und dem Naturschutz im Ergebnis nicht förderlich sind" oder die Grenze zum Querulatorischen beispielsweise durch eine Überhäufung der Staatsanwaltschaft mit Strafanzeigen aus nichtigen Anlässen oder sachfremden Erwägungen überschreitet. ${ }^{44}$ Demgegenüber lässt ein „,Drohen“ mit Schadensersatz- oder Amtshaftungsansprüchen oder Dienstaufsichtsbeschwerden als gesetzlich zulässigen Instrumenten zur Begegnung bewusst fehlerhaften Behördenhandelns oder das Vertreten unterschiedlicher wissenschaftlicher Positionen die Anerkennungsfähigkeit nicht entfallen. ${ }^{45}$ Zutreffend wies der VGH Kassel darauf hin, dass die anzuerkennenden Umweltverbände nicht als Unterstützer oder gar Verwaltungshelfer der Behörden fungieren, sondern ihnen die Wahrnehmung von Kontrollfunktionen hinsichtlich der Beachtung der Umweltbelange obliegt. ${ }^{46}$ Jüngst verneinte der VGH Mannheim einen Anspruch der Tierschutzorganisation PETA auf Anerkennung nach dem TierSchVKG BW, da aufgrund der nur sehr geringen Zahl von sieben ordentlichen Mitgliedern die Gewähr für eine sachgerechte Aufgabenerfüllung nicht hinreichend dauerhaft gesichert sei. ${ }^{47}$

Der EuGH entschied, dass die nationalen Rechtsvorschriften in Bezug auf die Nichtregierungsorganisationen einen weiten Gerichtszugang ermöglichen müssen und die praktische Wirksamkeit der Rechtsschutzregelungen in der UVP-Richtlinie nicht beeinträchtigen dürfen. Zwar könne zur Sicherstellung der tatsächlichen Existenz und Tätigkeit der Vereinigung eine Mindestanzahl an Mitgliedern verlangt werden, die jedoch nicht so hoch angesetzt werden darf, dass sie dem Ziel der einfachen Ermöglichung der gerichtlichen Kontrolle bestimmter Vorgänge zuwiderläuft. ${ }^{48}$ Auch lokalen Vereinigungen, die möglicherweise eher kleinere oder unbedeutendere Projekte anfechten, die aber dennoch erhebliche Umweltauswirkungen haben, muss ein Gerichtszugang eröffnet werden. ${ }^{49}$

\section{2 Überlegungen zur Änderung der Anerkennungsvoraussetzungen}

Mit Spannung bleibt die Weiterentwicklung etwaiger Änderungen der Anerkennungsvoraussetzungen zu beobachten. Im Februar 2019 brachte die AfD-Fraktion einen Gesetzentwurf zur erneuten Änderung des UmwRG ein, dessen Ziel in der Verschärfung der Anerkennungsvoraussetzungen liegt. ${ }^{50}$ Die aktuell niederschwelligen Voraussetzungen für die Anerkennung würden ein Missbrauchspotenzial in sich bergen. So könnten nur wenige Mitglieder umfassende Vereinigungen Zuwendungen von Unternehmen oder Organisationen erhalten, die im Interesse ausländischer Wettbewerber oder Staaten agieren, um deutschen Wirtschaftsunternehmen durch die Initiierung von Verbandsklagen zu schaden. Als Lösung wird vorgeschlagen, dass die Aner- kennung davon abhängig gemacht werden sollte, dass sich die Tätigkeit der Vereinigung ausschließlich auf Umweltschutzziele zu konzentrieren habe und insbesondere keinerlei kommerziellen Zwecke verfolgt würden. ${ }^{51}$ Hier tut sich erneut die Frage der Unionsrechtskonformität auf. In der Auslegungsmitteilung der Kommission zu den Umweltrechtsbehelfen stellte sich diese auf den Standpunkt, dass die bisherige EuGH-Rechtsprechung zur Anerkennung nicht so aufgefasst werden könne, dass die Nichtregierungsorganisation lediglich Umweltschutzziele verfolgen muss, aber verlangt werden dürfe, ,dass der Umweltschutz ein prioritäres oder wesentliches Ziel der NRO ist".52

Überdies ist ein Gesetzesvorschlag zur „Modernisierung des Gemeinnützigkeitsrechts" angekündigt. ${ }^{53} \mathrm{Da}$ die anzuerkennenden Vereinigungen nach $\$ 3$ Abs. 1 S. 1 Nr. 4 UmwRG gemeinnützige Zwecke verfolgen müssen, ${ }^{54}$ können sich hieraus beträchtliche Konsequenzen für das Recht der Umweltrechtsbehelfe ergeben. Wie brisant dieses Kriterium ist, zeigt sich anhand einer BFH-Entscheidung vom Januar 2019, die den attac-Trägerverein betraf und bei der der BFH herausarbeitete, dass gemeinnützige Körperschaften kein allgemeinpolitisches Mandat haben. Nach $\ 52$ Abs. 2 Nr. 24 AO ist die allgemeine Förderung des demokratischen Staatswesens als Förderung der Allgemeinheit anzuerkennen, allerdings fallen darunter nicht Bestrebungen, die nur bestimmte Einzelinteressen staatsbürgerlicher Art verfolgen. Infolgedessen darf weder ein politischer Zweck als alleiniger und ausschließlicher oder überwiegender Zweck in der Satzung festgelegt sein noch die Vereinigung mit ihrer tatsächlichen Geschäftsführung ausschließlich oder überwiegend einen politischen Zweck verfolgen. ${ }^{55}$ Unbedenklich sei dagegen, wenn eine steuer-

41) BT-Drs. 18/9526, S. 39; s. zum räumlichen Zusammenhang auch VG Sigmaringen, Urt. v. 14.11.2018 - 10 K 118/17, Rdnr. 89 , 92, juris; s. auch Decker, VBlBW 2018, 441, 445; Fellenberg/Schiller, in: Landmann/Rohmer, Umweltrecht, Stand: 6/2019, \$2 UmwRG Rdnr. 21; Happ, in: Eyermann, VwGO, 15. Aufl. 2019, $\$ 2$ UmwRG Rdnr. 6.

42) VG Wiesbaden, Beschl. v. 22. 8. 2019 - 4 L 2007/18.Wi, Rdnr. 44 juris; s. auch VGH Kassel, Beschl. v. 24.1.2019 - 9 B 2455/18, NVwZ-RR 2019, 770, 771.

43) VG Wiesbaden, Beschl. v. 22.8.2019 - 4 L 2007/18.Wi, Rdnr. 44, juris; s. auch VGH Kassel, Beschl. v. 24.1.2019 - 9 B 2455/18, NVwZ-RR 2019, 770, 771.

44) VG Wiesbaden, Beschl. v. 22. 8. 2019-4 L 2007/18.Wi, Rdnr. 45, juris.

45) VGH Kassel, Beschl. v. 24.1.2019 - 9 B 2455/18, NVwZ-RR 2019, 770, 771; VG Wiesbaden, Beschl. v. 22.8.2019 - 4 L 2007/18.Wi, Rdnr. 45, juris.

46) VGH Kassel, Beschl. v. 24.1.2019 - 9 B 2455/18, NVwZ-RR 2019, 770, 771 .

47) VGH Mannheim, Urt. v. 12.3.2020 - 1 S 720/18, Rdnr. 95 ff., juris.

48) EuGH, Urt. v. 15. 10.2009 - C-263/08, ECLI:EU:C:2009:63, Rdnr. 45, 47

49) Komm., Mitt. v. 28.4.2017, über den Zugang zu Gerichten in Umweltangelegenheiten, C(2017) 2616 final, S. 27.

50) BT-Drs. 19/7702.

51) BT-Drs. 19/7702, S. 2.

52) Komm., Mitt. v. 28.4.2017, über den Zugang zu Gerichten in Umweltangelegenheiten, C(2017) 2616 final, S. 26.

53) S. den Artikel von Janisch, „Ein Platz am Topf“, SZ v. 25.10.2019, Nr. 247, S. 7; s.a. den Artikel von Anger/Neuerer, Scholz will das Gemeinnützigkeitsrecht reformieren, Handelsblatt v. 22.10.2019, Stand 11.11.2019, abrufbar unter https:// www.handelsblatt.com/politik/deutschland/nach-campact-entscheidung-scholz-will-das-gemeinnuetzigkeitsrecht-reformieren /25142352.html?ticket $=$ ST $-89282491-$ eLRq2p0DocePbxRUeqFN-ap4.

54) Näher dazu Gröhn, NuR 2019, 225, 232.

55) BFH, Urt. v. 10.1.2019 - V R 60/70, GewArch 2019, 207, 208 unter Verweis auf BFH, Urt. v. 29.8.1984 - I R 203/81, Rdnr. 29, juris 
lich begünstigte Tätigkeit im Einzelfall zwangsläufig mit einer gewissen politischen Zielsetzung verbunden ist, etwa wenn eine Bürgerinitiative mit ihrer satzungsmäßigen Tätigkeit den Umweltschutz fördert, indem sie der objektiven Meinungsbildung als Grundlage zur Lösung der mit Entsorgungsvorhaben zusammenhängenden Umweltprobleme sowie der daraus resultierenden Ziel- und Interessenkonflikte dient. ${ }^{56}$ Außerdem muss die Vereinigung ,,parteipolitisch neutral“ agieren. Die in $\$ 52$ Abs. 2 Nr. 7 AO als Förderung der Allgemeinheit anzusehende Förderung der Erziehung, Volks- und Berufsbildung sei auf die Schaffung und Förderung politischer Wahrnehmungsfähigkeit und politischen Verantwortungsbewusstseins sowie die Diskussion politischer Fragen ,in geistiger Offenheit" gerichtet, gestatte jedoch nicht die Transportierung dermaßen entwickelter Ergebnisse durch Einflussnahme auf die politische Willensbildung und öffentliche Meinung. ${ }^{57}$

\subsection{Verfahren vor dem $A C C$}

Schließlich sei darauf aufmerksam gemacht, dass vor dem ACC ein Verfahren eingeleitet wurde, weil in Deutschland nur solche Vereinigungen anerkannt werden können, die jeder Person den Eintritt als Mitglied zur Unterstützung ermöglichen ( $\$ 3$ Abs. 1 S. 1 Nr. 5 UmwRG). Damit sind Vereinigungen ohne vollständig binnendemokratische Organisation, wie z.B. die Stiftung WWF und der eingetragene Verein Greenpeace, von einer Anerkennung ausgeschlossen. In einer aktuellen, jedoch das TierSchVKG BW betreffenden Entscheidung bemängelte der VGH Mannheim in Bezug auf die Tierschutzorganisation PETA, dass diese auf ihrer Homepage die Fördermitgliedschaft so sehr in den Vordergrund stelle, dass Interessenten nur mit erheblichem Aufwand erfahren können, dass es auch die Möglichkeit der ordentlichen Mitgliedschaft mit Stimmrecht gebe, welche Rechte und Pflichten mit dieser verbunden seien und ein Antragsformular für eine solche nicht zu finden sei. ${ }^{58}$ In dem Verfahren vor dem ACC wird geltend gemacht, dass diese Anforderung unverhältnismäßig sei und zu Diskriminierungen im Vergleich zu ausländischen Vereinigungen führe. ${ }^{59}$ In seiner Erwiderung verwies Deutschland darauf, dass die AK gerade keine actio popularis verlange und das einengende Erfordernis darauf zurückgehe, dass sich bei einem demokratischen Ansatz die Legitimation aus der Beteiligung von Bürgern ergebe und die AK sich selbst der „environmental democracy“ verschrieben habe. ${ }^{60}$ Jüngst stellte sich der VGH Mannheim, allerdings bezogen auf das TierSchVKG BW, auf den Standpunkt, dass sich der eine Ungleichbehandlung rechtfertigende Grund aus dem Anliegen ergebe, ,dass die anerkannten Tierschutzorganisationen sich als unabhängige, möglichst plural zusammengesetzte Sachwalter der Allgemeinheit für den Tierschutz einsetzen und daher Vereinigungen, die sich nur einem eingeengten, einseitig zusammengesetzten Kreis von Interessenten öffnen, nicht anerkannt werden sollen“" ${ }^{61}$ Sollen Tierschutzorganisationen sozusagen als „Anwalt der Tiere“ Gemeinwohlinteressen wahrnehmen, müssten sie intern so verfasst sein, dass sie auch die Allgemeinheit repräsentieren. ${ }^{62}$ Die EU-Kommission wies in ihrer davon unabhängig erlassenen Auslegungsmitteilung darauf hin, dass nicht alle klagebefugten Nichtregierungsorganisationen in den Mitgliedstaaten mitgliederbasiert sind und z.B. manche Klagen gemeinnütziger Stiftungen zu mitunter bedeutenden EuGH-Entscheidungen geführt hätten. ${ }^{63}$ Während demnach einige andere Mitgliedstaaten sich bei der Anerkennung der NGOs mit einem funktionalistischen Leitbild begnügen und ausschließlich auf das Einbringen ihrer Expertise setzen, tritt in Deutschland als weitere Voraussetzung für die Anerkennung das Demokratisierungsleitbild hinzu. ${ }^{64} \mathrm{Da}$ die Mitgliedstaaten nach Art. 9 Abs. $3 \mathrm{AK}$ in ihrem innerstaatlichen Recht die von den Mitgliedern der Öffentlichkeit zu erfüllenden Kriterien für Umwelt- rechtsbehelfe festlegen dürfen, spricht die daraus resultierende Gestaltungsfreiheit an und für sich dafür, dass die Anerkennungsvoraussetzungen in den einzelnen Mitgliedstaaten durchaus variieren können. Problematisch dürfte die Rechtslage erst werden, wenn die Anerkennungsvoraussetzungen so eng gezurrt werden, dass der von der AK angestrebte effektive Umweltrechtsschutz zu stark beeinträchtigt wird.

\section{Differenziertes Vorgehen in Bezug auf die materielle Verbandspräklusion}

Nachdem der EuGH die materielle Präklusion in $\$ 2$ Abs. 3 S. 1 UmwRG sowie in $\$ 73$ Abs. 4 VwVfG für unvereinbar mit Art. 11 UVP-Richtlinie 2011/92/EU und Art. 25 IERichtlinie 2010/75/EU erklärt hatte, ${ }^{65}$ wurde im Zuge der UmwRG-Novelle $\$ 2$ Abs. 3 S. 1 UmwRG aufgehoben. In Reaktion auf die Entscheidung des EuGH sowie den Beschluss V/9h der 5 . Vertragsstaatenkonferenz zur AK ${ }^{66}$ bestimmt nunmehr $\$ 7$ Abs. 4 UmwRG, dass im Rechtsbehelfsverfahren gegen eine Entscheidung nach $\$ 1$ Abs. $1 \mathrm{~S} .1$ Nrn. 1-2b die Regelungen des $\$ 73$ Abs. 4 S. 3-6 VwVfG, auch in den Fällen seines Absatzes 8, keine Anwendung finden. ${ }^{67}$ Anders ausgedrückt dürfen die sich erst im Gerichtsverfahren auswirkenden materiellen Präklusionsregeln nicht mehr angewendet werden, bleibt aber die vor allem der Entlastung des Verwaltungsverfahrens dienende formelle Präklusion erhalten. ${ }^{68}$ Von Letzterer sollten die Behörden aber zurückhaltend Gebrauch machen, da prä-

56) BFH, Urt. v. 10.1.2019 - V R 60/70, GewArch 2019, 207, 208 57) BFH, Urt. v. 10.1.2019 - V R 60/70, GewArch 2019, 207, 209.

58) VGH Mannheim, Urt. v. 12.3.2020 - 1 S 720/18, Rdnr. 107 ff., juris.

59) S. Mitteilung WWF Deutschland an das Compliance Committee v. 10.2.2016, zugleich Einleitung des Verfahrens ACCC/ C/2016/137 Germany, Stand 11.11.2019, abrufbar unter https:// www.unece.org/fileadmin/DAM/env/pp/compliance/C2015137_Germany/Communication_Germany_WWF_resubmitted_10.2.2016.pdf, S. 7f.; es ist fraglich, ob das VG Berlin, Urt. v. 31.10.2019-10 K 412.18, Rdnr. 86 ff., juris, ohne sich mit der fehlenden Anerkennung von Greenpeace eingehender auseinanderzusetzen, damit eine bewusste Aussage treffen wollte, da es schwerpunktmäßig auf die fehlende Klagebefugnis abstellte. S. dazu auch Gröhn, NuR 2019, 225, 232 f., wonach diese Anforderung den Zielen der Stärkung des Naturschutzes, der Gewährleistung breiter Beteiligungsmöglichkeiten sowie eines weiten Gerichtszugangs zuwiderlaufe.

60) S. einleitende Stellungnahme Deutschlands bei der Anhörung vor dem 61. Treffen des Compliance Committees betreffend des Verfahrens ACCC/C/2016/137 Germany, Stand 11.11.2019, abrufbar unter https://www.unece.org/fileadmin/DAM/env/pp/ compliance/C2015-137_Germany/Correspondence_with_the_ Party_concerned/frPartyC137_opening_statement_for_hearing_at_CC61.pdf, S. $6 \mathrm{f}$.

61) VGH Mannheim, Urt. v. 12.3.2020 - 1 S 720/18, Rdnr. 104

62) VGH Mannheim, Urt. v. 12.3.2020 - 1 S 720/18, Rdnr. 102.

63) Komm., Mitt. v. 28.4.2017, über den Zugang zu Gerichten in Umweltangelegenheiten, C(2017) 2616 final, S. 27.

64) $\mathrm{Zu}$ den verschiedenen Leitbildern hinter NGOs auf der völkerrechtlichen Ebene s. v. Bernstorff, VVDStRL 79, 2020, 381, $387 \mathrm{ff}$.

65) EuGH, Urt. v. 15.10.2015 - C-137/14, ECLI:EU:C:2015:683, Rdnr. $75 \mathrm{ff}$.

66) BT-Drs. 18/9526, S. 44; s. auch Schlacke, NVwZ 2019, 1392, 1393.

67) S. auch $₫ 18$ Abs. 1 S. 3 UVPG, in dem nun nicht mehr auf $₫ 73$ Abs. 4 VwVfG verwiesen wird. Zur formellen Präklusion Guckelberger, Deutsches Verwaltungsprozessrecht unter unionsrechtlichem Anpassungsdruck, 2017, S. 195 ff.; Siegel, NVwZ 2016, 337, $338 \mathrm{f}$.

68) Durner, Rechtsgutachten zur Wiedereinführung der Präklusion v. 7.10.2019, Stand 16.12.2019, abrufbar unter https://www. netzwerk-bahnen.de/assets/files/news/2019/2019_10_07_durner_rechtsgutachten_wiedereinfuehrung-der-praeklusion.pdf, S. 43; s. auch Széchényi, BayVBl. 2016, 366, 369 f. 
kludierte Einwendungen durchaus entscheidungsrelevante Informationen enthalten können. ${ }^{69}$

\subsection{Gesetzgebungskompetenz des Bundes in Bezug auf $\$ 7$ Abs. 4 UmwRG}

Man kann darüber streiten, ob sich die Regelung des $\$ 7$ Abs. 4 UmwRG nur auf die Vorschriften des VwVfG des Bundes oder auch die korrespondierenden Vorschriften im Landesrecht bezieht. Da der Bundesgesetzgeber mit der UmwRGNovelle eine vollständige europarechtskonforme Umsetzung des Umweltrechtsschutzes anstrebte, judizierte das BVerwG, dass diese Vorschrift so zu verstehen ist, dass davon auch gleichlautende landesrechtliche Regelungen erfasst werden. Auch in weiteren Vorschriften des UmwRG, z. B. \$4 Abs. 1a S. 1, Abs. 1b Nrn. 1, 2 UmwRG, werde nicht zwischen den Verwaltungsverfahrensgesetzen von Bund und Ländern differenziert. ${ }^{70}$ Wäre weiterhin von der Einschlägigkeit des $\$ 43 \mathrm{a}$ Nr. 7 EnWG a. F. auszugehen, sei $\$ 7$ Abs. 4 UmwRG auf diese Vorschrift im Wege der Analogie anzuwenden. ${ }^{71}$

Zwar kann man Zweifel an der Gesetzgebungskompetenz des Bundes hinsichtlich der „Beseitigung“ materieller Präklusionsnormen der Länder aufwerfen, zumal man derartige Regelungen auch dem materiellen Recht zuordnen kann. ${ }^{72}$ Stellt man sich nämlich auf den Standpunkt, dass die Präklusion zum Ausschluss bzw. zur Vernichtung subjektiver Rechte führt, würde es sich hierbei um gar keine das gerichtliche Verfahren betreffende prozessuale Regelungen handeln. ${ }^{73}$ Dieser an subjektive Rechte anknüpfende Ansatz passt aber jedenfalls nicht auf davon losgelöste Verbandsrechtsbehelfe. ${ }^{74}$ Da die materiellen Präklusionsnormen ihre eigentlichen Konsequenzen im gerichtlichen Verfahren durch den Ausschluss von Einwendungen zeitigen ${ }^{75}$ und der EuGH bei seiner Entscheidung, auf welche der UmwRGGesetzgeber reagieren wollte, auf die damit verbundene Beschränkung der Rechtsbehelfe und nicht auf das Verwaltungsverfahren abstellte, ${ }^{76}$ kann man ein solches Vorgehen durchaus noch mit der Gesetzgebungskompetenz des Bundes für das gerichtliche Verfahren aus Art. 74 Abs. 1 Nr. 1 GG in Einklang bringen. ${ }^{77}$ Indem der Gesetzgeber gem. $\$ 7$ Abs. 4 UmwRG nur bestimmt, dass die bezeichneten verwaltungsverfahrensgesetzlichen Präklusionsregelungen keine Anwendung finden, d.h. für unanwendbar erklärt werden, enthält diese Vorschrift eine nur auf das gerichtliche Verfahren bezogene und damit der Gesetzgebungskompetenz des Bundes unterfallende Regelung. ${ }^{78}$ Weil die EuGH-Rechtsprechung im aufgezeigten Umfang sinngemäß auf vergleichbare materielle Präklusionsnormen von Bund und Ländern übertragbar ist, ${ }^{79}$ müssten bei Bejahung einer fehlenden Gesetzgebungskompetenz des Bundes jedenfalls die entsprechenden Landesnormen kraft Unionsrechts unangewendet bleiben. ${ }^{80}$

Nur der Vollständigkeit halber sei darauf aufmerksam gemacht, dass $\$ 7$ Abs. 4 UmwRG nicht nur für Rechtsbehelfe von Umweltvereinigungen gilt, sondern gem. $\$ 7$ Abs. 6 UmwRG auch auf Rechtsbehelfe von natürlichen wie juristischen Personen und Vereinigungen nach $₫ 4$ Abs. 3 S. 1 Nr. 1 UmwRG Anwendung findet. Die Nichtanwendbarkeit der materiellen Präklusion greift nach dem Gesetzeswortlaut des $\$ 7$ Abs. 4 UmwRG auch bei Einwendungen ohne unmittelbaren Umweltbezug. ${ }^{81}$ Eine Differenzierung würde an den Unionsrichtlinien und an Art. 9 Abs. 2 AK scheitern. ${ }^{82}$

\subsection{Beibehaltung der materiellen Präklusion bei SUP-pflichtigen Plänen und Programmen}

Da die materielle Präklusion in Deutschland eine beliebte Rechtsfigur ist, für die es - wie auch in der Rechtssache Protect dargelegt - gute Argumente gibt, entschied man sich bei den SUP-pflichtigen Plänen und Programmen für deren grundsätzliche Beibehaltung. So wurde für diese eine dem bisherigen $\$ 2$ Abs. 3 UmwRG entsprechende Präklusionsregelung in $\$ 7$ Abs. 3 UmwRG eingeführt. ${ }^{83}$ Danach ist eine
Vereinigung i.S.d. $₫ 4$ Abs. 3 S. 1 Nr. 2 UmwRG, die in einem Verfahren nach $\$ 1$ Abs. 1 S. 1 Nr. 4 UmwRG Gelegenheit zur Äußerung hatte, im Umweltrechtsbehelfsverfahren mit allen Einwendungen ausgeschlossen, die sie im Verfahren nicht oder nach den geltenden Rechtsvorschriften nicht rechtzeitig geltend gemacht hat, obwohl sie diese hätte geltend machen können. $\$ 7$ Abs. 3 S. 2 UmwRG nimmt von dieser materiellen Präklusion die Aufstellung, Änderung, Ergänzung oder Aufhebung von Bebauungsplänen nach \$10 BauGB aus. Begründet wurde diese Ausnahme damit, dass die Unterscheidung zwischen UVP- und SUP-pflichtigen Bebauungsplänen der Praxis Probleme bereitet. ${ }^{84}$ Zutreffend weist jedoch Mager darauf hin, dass den Vereinigungen eine solche Prüfung aufgrund der Anforderungen des $\$ 2$ Abs. 1 S. 1 Nr. 3 lit. b) UmwRG nicht erspart wird. ${ }^{85}$ Infolgedes-

69) Durner, Rechtsgutachten zur Wiedereinführung der Präklusion v. 7.10.2019, Stand 16.12.2019, abrufbar unter https://www. netzwerk-bahnen.de/assets/files/news/2019/2019_10_07_durner_rechtsgutachten_wiedereinfuehrung-der-praeklusion.pdf, S. 43 unter Verweis auf BVerwG, Urt. v. 11. 10.2017 - 9 A 14/16, NVwZ-Beilage 2018, 41, 42 Rdnr. 16.

70) BVerwG, Urt. v. 14.12.2017 - 4 C 6/16, NuR 2018, 267, 268; s. auch Berkemann, DVB1. 2020, 1, 12

71) BVerwG, Urt. v. 14.12.2017 - 4 C 6/16, NuR 2018, 267, 268 f.

72) So auch BVerwG, Beschl. v. 12.1.2018 - 9 A 12.17, 9 A 3.17, DVB1. 2018, 585, 586; s. auch Durner, Rechtsgutachten zur Wiedereinführung der Präklusion v. 7.10.2019, Stand 16.12.2019, abrufbar unter https://www.netzwerk-bahnen.de/assets/files/ news/2019/2019_10_07_durner_rechtsgutachten_wiedereinfuehrung-der-praeklusion.pdf, S. $55 \mathrm{f}$.

73) $\mathrm{Zu}$ dieser Sichtweise, sie aber letztlich ablehnend Oexle, Das Rechtsinstitut der materiellen Präklusion in den Zulassungsverfahren des Umwelt- und Planungsrechts, 2000, S. $19 \mathrm{ff}$.

74) Wie hier, aber bezogen auf die Jedermanns-Öffentlichkeitsbeteiligung, Oexle, Das Rechtsinstitut der materiellen Präklusion in den Zulassungsverfahren des Umwelt- und Planungsrechts, 2000, S. 22f. Dazu, dass es bei der materiellen Präklusion um die Einbeziehung Drittbetroffener in das Verwaltungsverfahren geht, die nicht unbedingt ein Interesse am Zustandekommen der Entscheidung haben, und daher eine Verwirkung von Rechten ablehnend Röhl/Ladenburger, Die materielle Präklusion im raumbezogenen Verwaltungsrecht, 1997, S. $20 \mathrm{ff}$., 48.

75) Röhl/Ladenburger, Die materielle Präklusion im raumbezogenen Verwaltungsrecht, 1997, S. 47 verorten diese im Schnittpunkt des Verfahrens-, materiellen und Prozessrechts.

76) Zur Argumentation des EuGH Jachmann, Das Ende der materiellen Präklusion, 2019, S. $12 \mathrm{f}$.

77) Zu Art. 74 Abs. 1 Nr. 1 GG und der dann aufzuwerfenden Frage, inwieweit der Bund von seiner Gesetzgebungskompetenz abschließenden Gebrauch gemacht hat, Niedzwicki, Präklusionsvorschriften des öffentlichen Rechts im Spannungsfeld zwischen Verfahrensbeschleunigung, Einzelfallgerechtigkeit und Rechtsstaatlichkeit, 2007, S. $214 \mathrm{ff}$. S. auch Oexle, Das Rechtsinstitut der materiellen Präklusion in den Zulassungsverfahren des Umweltund Planungsrechts, 2000, S. $108 \mathrm{ff}$.

78) Zur Unanwendbarkeit Durner, Rechtsgutachten zur Wiedereinführung der Präklusion v. 7. 10.2019, Stand 16.12.2019, abrufbar unter https://www.netzwerk-bahnen.de/assets/files/news/2019/ 2019_10_07_durner_rechtsgutachten_wiedereinfuehrung-derpraeklusion.pdf, S. $16 \mathrm{f}$.

79) Siegel, NVwZ 2016, 337, 340.

80) So auch die Position von Durner, Rechtsgutachten zur Wiedereinführung der Präklusion v. 7.10.2019, Stand 16.12.2019, abrufbar unter https://www.netzwerk-bahnen.de/assets/files/ news/2019/2019_10_07_durner_rechtsgutachten_wiedereinfuehrung-der-praeklusion.pdf, S. 56.

81) OVG Bautzen, Urt. v. 27.11.2018 - 4 C 22/17, Rdnr. 30, juris.

82) OVG Bautzen, Urt. v. 27.11.2018 - 4 C 22/17, Rdnr. 30, juris (ohne Erwähnung der AK); Jachmann, Das Ende der materiellen Präklusion, 2019, S. 17 f.; Schlacke, NVwZ 2019, 1392, 1394; Siegel, NVwZ 2016, 337, 339.

83) BT-Drs. 18/9526, S. 43.

84) BT-Drs. 18/9526, S. 43; s. auch Arndt, UPR 2018, 90, 91; Bunge, in: ders., UmwRG, 2. Aufl. 2019, \$7 Rdnr. 65.

85) Mager, EurUP 2018, 50, 55. 
sen kommt die materielle Präklusion bei Raumordnungs- ${ }^{86}$ oder Flächennutzungsplänen (siehe auch $\$ 3$ Abs. 3 BauGB) zum Tragen. ${ }^{87}$ Obwohl man durchaus Bedenken an der Vereinbarkeit dieser Norm mit Art. 9 Abs. 3 AK anmelden $\mathrm{kann}^{88}$ und sich auch die Kommission auf den Standpunkt gestellt hat, dass die EuGH-Rechtsprechung zu Art. 9 Abs. 2 AK sinngemäß auch für Art. 9 Abs. 3 AK gilt, dürfte der EuGH bei Letzterem nach seiner Protect-Rechtsprechung einer materiellen Präklusion nicht per se ablehnend gegenüberstehen. ${ }^{89}$ Offen bleibt aber, ob dem deutschen Gesetzgeber eine verhältnismäßige Ausgestaltung der verschiedenen konfligierenden Umweltrechtsschutzparameter (siehe Art. 9 Abs. 4 AK) gelungen ist. ${ }^{90}$

\subsection{Unterschiedliche Bewertung der Folgen der Zurückführung}

Die Folgen der Zurückführung der materiellen Präklusion im Umweltrechtsschutz für die Gerichtsverfahren werden unterschiedlich eingeschätzt. Nach Meinung von $R u$ bel lässt sich eine verzögernde Wirkung nicht ausschließen, eine solche sei jedoch bislang in der Gerichtspraxis nicht augenfällig geworden. Die früher gebotene Klärung des Präklusionseintritts sei demgegenüber teils streitanfällig und zeitaufwendig gewesen. ${ }^{91}$ Auch sei die Annahme, Umweltverbände würden nun Einwendungen bewusst zurückhalten anstelle ihre frühzeitigen Einflussnahme-Chancen auf das Vorhaben während des Planfeststellungsverfahrens zu nutzen, fraglich. ${ }^{92}$ Es zeichne sich jedoch nach seiner Meinung die Entwicklung ab, dass Verbände bei Verbandsklagen eine Art Stellvertreterfunktion für Individualkläger übernehmen. ${ }^{93}$ Anstelle mehrerer Individualklagen lässt sich als Vorteil solcher Verbandsklagen aber immer noch deren Bündelungsfunktion ausmachen. Durner berichtet von Missbrauchsfällen, in denen ein Verband zur Verhinderung von Konkurrenz gegründet worden sei. ${ }^{94}$ Nach seiner Einschätzung ist eine komplette Einstellung der Mitwirkung der Verbände im Verfahren nicht zu befürchten, aber bestehe das schwer abzuschätzende Risiko, dass diese künftig nicht oder jedenfalls weniger intensiv an Beteiligungsverfahren mitwirken werden, wenn eine Klage aufgrund prinzipieller Ablehnung eines Projekts wahrscheinlich ist. ${ }^{95}$ Andere Stimmen beklagen das aus richterlicher Sicht unerfreuliche Verzögerungspotenzial, da sich die Befassung mit möglichen umweltrechtlichen Verstößen hin zu den verwaltungsgerichtlichen Tatsacheninstanzen verlagern und damit die funktionsgerechte Aufgabenverteilung zwischen Verwaltung und Gerichtsbarkeit gefährden würde. ${ }^{96}$ Deshalb hat sich die 89. Justizministerkonferenz am 15.11.2018 dafür ausgesprochen, die $\mathrm{AK}$ und das einschlägige Unionsrecht dergestalt anzupassen, dass die materielle Präklusion und die Beschränkungen des gerichtlichen Prüfumfangs auf umweltbezogene Rechtsvorschriften wieder umfassend in das UmwRG aufgenommen werden können. ${ }^{97}$ Ein solcher Weg benötigt jedoch Zeit und müsste erst bei den anderen Mitgliedstaaten bzw. Vertragsparteien auf Zustimmung stoßen.

\section{Missbräuchliches oder unredliches Verhalten im Rechtsbehelfsverfahren, $\$ 5$ UmwRG}

Da der EuGH in seiner Entscheidung zur Unzulässigkeit materieller Präklusionsvorschriften im Anwendungsbereich des Art. 9 Abs. 2 AK zugleich die Möglichkeit der Zurückweisung von unredlichem oder missbräuchlichem Vorbringen gebilligt hat, wurden seine Ausführungen fast wortgleich in das UmwRG übernommen. ${ }^{98} \mathrm{Gem}$. $\$ 5$ UmwRG bleiben Einwendungen, die erstmals im Rechtsbehelfsverfahren erhoben werden, unberücksichtigt, sofern die erstmalige Geltendmachung missbräuchlich oder unredlich ist. Unter derartige „Einwendungen“ fällt erstmals im Rechtsbehelfsverfahren geltend gemachtes sachliches Gegenvorbringen; Ausführungen zum Rechtsrahmen sind dagegen stets möglich. ${ }^{99}$ Die sich sowohl auf Individualkläger als auch auf Umweltschutzvereinigungen beziehende Vorschrift ist nicht ohne Kritik geblieben, welche sich vor allem darauf stützt, dass mangels Regelbeispielen die Verantwortung zur Konkretisierung der unbestimmten Rechtsbegriffe der Praxis aufgebürdet wurde. ${ }^{100}$ Andererseits wäre die Aufstellung von Regelbeispielen mit dem Risiko verbunden gewesen, dass einzelne Fallgruppen möglicherweise doch nicht dem Unionsrecht standhalten. ${ }^{101}$ Aus $\$ 5$ UmwRG geht hinreichend deutlich hervor, dass nur ein erheblicher Vorwurf hinsichtlich des Verhaltens des Rechtsbehelfsführers die Folgen des $\$ 5$ UmwRG auszulösen vermag. ${ }^{102}$ Die Gerichte können die Vorschrift im Einzelfall unter Beachtung des Unionsrechts angemessen justieren. ${ }^{103}$ Sie haben

86) Mager, EurUP 2018, 50, 55; Schlacke, NVwZ 2019, 1392, 1395.

87) Arndt, UPR 2018, 90, 95; Mager, EurUP 2018, 50, 55; Schlacke, NVwZ 2019, 1392, 1395

88) Jachmann, Das Ende der materiellen Präklusion, 2019, S. 24f.; s. auch Buchberger, EurUP 2019, 377, 384; Decker, VBlBW 2018, $441,445$.

89) Bunge, in: ders., UmwRG, 2. Aufl. 2019, \$7 Rdnr. 11; Komm., Mitt. v. 28. 4.2017, über den Zugang zu Gerichten in Umweltangelegenheiten, C(2017) 2616 final, Rdnr. 121.

90) Wohl im Hinblick auf Letzteres zweifelnd Franzius, NuR 2019, 649, 652.

91) Rubel, EurUP 2019, 386, 394. Dazu, dass die materielle Präklusion sich nicht spürbar ausgewirkt hat, $W y$ sk, in: Appel/Wagner-Kardenal, Verwaltung zwischen Gestaltung, Transparenz und Kontrolle, 2019, S. 27, 42.

92) Rubel, EurUP 2019, 386, 394.

93) Rubel, EurUP 2019, 386, 387 f.; s. auch Külpmann, DVB1. 2019, 140, 142.

94) Durner, EurUP 2018, 142, 155

95) Durner, Rechtsgutachten zur Wiedereinführung der Präklusion v. 7.10.2019, Stand 16.12.2019, abrufbar unter https://www. netzwerk-bahnen.de/assets/files/news/2019/2019_10_07_durner_rechtsgutachten_wiedereinfuehrung-der-praeklusion.pdf, S. 38; s. auch Zeissler/Schmitz, UPR 2016, 1, 3.

96) Storost, UPR 2018, 52, 56; s. auch Geiger/Mader, GewArch 2017, $366,370$.

97) JuMikO, Herbstkonferenz v. 15.11.2018, TOP I.7 unter 2.

98) BT-Drs. 18/9526, S. 41. Dazu, dass es sinnvoll war, sich eng an die Vorgaben des EuGH anzulehnen, Durner, Rechtsgutachten zur Wiedereinführung der Präklusion v. 7.10.2019, Stand 16.12.2019, abrufbar unter https://www.netzwerk-bahnen.de/ assets/files/news/2019/2019_10_07_durner_rechtsgutachten_ wiedereinfuehrung-der-praeklusion.pdf, S. 44.

99) OVG Hamburg, Beschl. v. 8.1.2020 - 2 Bs 183/19, Rdnr. 38, juris.

100) Schlacke, NVwZ 2017, 905, 910 (stellt dies nur fest, äußert aber keine Kritik); Siegel, ZUR 2019, 451, 454f., der darüber hinaus kritisiert, dass der vom EuGH anerkannte Gestaltungsspielraum der Mitgliedstaaten nicht genutzt wurde. Dazu, dass durch die wörtliche Übernahme keine Bedenken an der Unionsrechtskonformität bestehen, Grandjot, in: Jan Hoffmann, 15 Jahre Zentrum für Rechts- und Verwaltungswissenschaften an der Brandenburgischen Technischen Universität, 2017, S. 137, 140, der auf S. 144 meint, dass diese Regelung angesichts des Grundsatzes von Treu und Glauben entbehrlich gewesen wäre. Ablehnend gegenüber der zuletzt genannten Position Fellenberg/Schiller, in: Landmann/Rohmer, Umweltrecht, Stand: 6/2019, $\$ 5$ UmwRG Rdnr. 10

101) Durner, Rechtsgutachten zur Wiedereinführung der Präklusion v. 7.10.2019, Stand 16.12.2019, abrufbar unter https://www. netzwerk-bahnen.de/assets/files/news/2019/2019_10_07_durner_rechtsgutachten_wiedereinfuehrung-der-praeklusion.pdf, S. 44; Klinger, ZUR 2016, 41, 42f.

102) Durner, Rechtsgutachten zur Wiedereinführung der Präklusion v. 7.10.2019, Stand 16.12.2019, abrufbar unter https://www.netzwerk-bahnen.de/assets/files/news/2019/2019_10_07_durner_ rechtsgutachten_wiedereinfuehrung-der-praeklusion.pdf, S. 44; s. auch Bunge, in: ders., UmwRG, 2. Aufl. 2019, \5 Rdnr. 3.

103) Durner, Rechtsgutachten zur Wiedereinführung der Präklusion v. 7.10.2019, Stand 16.12.2019, abrufbar unter https://www.netzwerk-bahnen.de/assets/files/news/2019/2019_10_07_durner_ rechtsgutachten_wiedereinfuehrung-der-praeklusion.pdf, S. 44. 
bezogen auf den jeweiligen Einzelfall das Vorliegen eines missbräuchlichen oder unredlichen Verhaltens zu prüfen. ${ }^{104}$

Allein aus dem Umstand, dass eine Vereinigung während des Planfeststellungsverfahrens untätig bleibt, lässt sich nach zutreffender Rechtsprechung aber im Umkehrschluss zu $\$ 2$ Abs. 1 Nr. 3 lit. a) UmwRG kein solches Verhalten entnehmen. ${ }^{105} \mathrm{Da}$ der EuGH die materielle Präklusion für unionsrechtswidrig hielt, ist $\$ 5$ UmwRG unionsrechtskonform eng auszulegen, um eine materielle Präklusion durch die Hintertür $\mathrm{zu}$ verhindern. ${ }^{106}$ Insoweit ist hinsichtlich der Heranziehung der Gesetzesmaterialien, nach denen die erstmalige Geltendmachung bestimmter Einwendungen, die der Vereinigung bereits im Zulassungsverfahren bekannt waren, als missbräuchlich angesehen werden könne, Vorsicht geboten, wenn sich die Vereinigung gemessen an den Zielen ihrer Satzung oder ihrer Rolle als ,Quasi-Verwaltungshelfer" sozusagen unvernünftig verhält. ${ }^{107}$ So wies auch das OVG Hamburg in Anlehnung an das Schrifttum darauf hin, dass die Verbände nicht als Quasi-Verwaltungshelfer zur Mitwirkung im Verfahren verpflichtet seien, sondern ihnen diese Stellung erst im Falle der Mitwirkung zukommt. ${ }^{108}$ Diese Argumentation erweist sich auch nicht im Hinblick auf $\$ 18$ Abs. 1 S. 3 UVPG als hinfällig, wonach die Vereinigungen die zuständige Behörde in einer dem Umweltschutz dienenden Weise unterstützen sollen, da andernfalls ein Widerspruch zur völker- und unionsrechtlichen Funktion der Umweltrechtsbehelfe von Verbänden entstünde. ${ }^{109}$

In den Worten des OVG Hamburg mag es missbräuchlich sein, wenn die Vereinigung während des Verwaltungsverfahrens nach außen deutlich zu verstehen gab, dass keine Einwände bestünden, solche dann aber, ohne ,klüger“ geworden zu sein, im gerichtlichen Verfahren erhebt. ${ }^{110} \mathrm{Da}-$ gegen ließ das OVG Lüneburg offen, ob man ein solches Verhalten auch konkludent aus einer Gesamtschau des Verhaltens während des Verfahrens entnehmen könne, und für eine unzulässige Rechtsausübung eine subjektive Komponente, etwa ein arglistiges Verschweigen, erforderlich sei. ${ }^{111}$ Letzteres ist zu bejahen. ${ }^{112}$ Nach dem OVG Münster setzt ein missbräuchliches oder unredliches Verhalten mehr als ein bloßes Schweigen auf eine Anfrage voraus, insbesondere wenn diese bloß informatorisch mit der Gelegenheit zur Äußerung ohne ausdrückliche Aufforderung zur Stellungnahme zugesendet wurde. ${ }^{113}$ Soweit das Erfordernis der Geltendmachung einer Verletzung umweltbezogener Rechtsvorschriften aufgehoben wurde, kann der Vortrag (auch) nichtumweltbezogener Rechtsverstöße durch eine Umweltschutzvereinigung nicht als missbräuchlich oder unredlich bewertet werden. ${ }^{114}$ Bei Verfahren, die wie das immissionsschutzrechtliche Genehmigungsverfahren nach $\int 19$ BImSchG keine Öffentlichkeitsbeteiligung kennen, bleibt $\$ 5$ UmwRG ohne Relevanz. ${ }^{115}$

Alles in allem zeichnet sich ab, dass der als Ausnahmevorschrift konzipierte $\$ 5$ UmwRG entsprechend seiner Natur nur selten greifen wird. ${ }^{116}$ Die Beweislast dafür liegt nicht bei den Vereinigungen, sondern obliegt den staatlichen Stellen. ${ }^{117}$ In der Praxis dürfte ein solcher Nachweis angesichts der subjektiven Komponente des $\int 5$ UmwRG nur schwer zu führen sein. ${ }^{118}$ Teilweise nehmen die Gerichte nicht abschließend zu dieser Vorschrift Stellung. Wegen der unionsrechtlichen Implikationen sollten sie sich bei der Auslegung auch an Aussagen der Unionsgerichte zu vergleichbaren Problemlagen orientieren. ${ }^{119}$

\section{Fazit}

$\int 5$ UmwRG kann aufgrund seiner engen Fassung die Folgen der Zurückführung der materiellen Präklusion kaum kompensieren. Alles in allem zeichnet sich der Trend zu Vorschlägen $a b$, die Rechtsschutzmöglichkeiten für Umweltschutzvereinigungen weiter einzuschränken. Diesen setzen jedoch das Unionsrecht und die AK Grenzen.
Open Access. Dieser Artikel wird unter der Creative Commons Namensnennung 4.0 International Lizenz veröffentlicht, welche die Nutzung, Vervielfältigung, Bearbeitung, Verbreitung und Wiedergabe in jeglichem Medium und Format erlaubt, sofern Sie den/die ursprünglichen Autor(en) und die Quelle ordnungsgemäß nennen, einen Link zur Creative Commons Lizenz beifügen und angeben, ob Änderungen vorgenommen wurden.

Die in diesem Artikel enthaltenen Bilder und sonstiges Drittmaterial unterliegen ebenfalls der genannten Creative Commons Lizenz, sofern sich aus der Abbildungslegende nichts anderes ergibt. Sofern das betreffende Material nicht unter der genannten Creative Commons Lizenz steht und die betreffende Handlung nicht nach gesetzlichen Vorschriften erlaubt ist, ist für die oben aufgeführten Weiterverwendungen des Materials die Einwilligung des jeweiligen Rechteinhabers einzuholen. Weitere Details zur Lizenz entnehmen Sie bitte der Lizenzinformation auf http://creativecommons.org/licenses/by/4.0/deed.de.

Open access funding provided by Projekt DEAL.

104) BT-Drs. 18/9526, S. 41; OVG Lüneburg, Urt. v. 31.7.2018 7 KS 17/16, Rdnr. 166, juris; Guckelberger Deutsches Verwaltungsprozessrecht unter unionsrechtlichem Anpassungsdruck, 2017, S. $199 \mathrm{f}$.

105) OVG Lüneburg, Urt. v. 15. 11.2018 - 1 KN 29/17, NVwZ-RR 2019, 631, 632; s. auch OVG Hamburg, Beschl. v. 15.8.2018 1 Es 1/18.P, ZUR 2019, 37, 39; Franzius, NuR 2019, 649, 653; in diese Richtung, aber doch zu einer Bejahung des $\$ 5$ UmwRG unter engen Voraussetzungen neigend, Fellenberg/Schiller Fellenberg/Schiller, in: Landmann/Rohmer, Umweltrecht, Stand: 6/2019, §5 UmwRG Rdnr. $23 \mathrm{f}$.

106) OVG Hamburg, Beschl. v. 15.8.2018 - 1 Es 1/18.P, ZUR 2019, 37, 39; Brigola/Heß, NuR 2017, 729, 732; Franzius, NuR 2019, 649, 653; s. auch Schlacke, NVwZ 2019, 1392, 1395.

107) BT-Drs. 18/9526, S. 41; kritisch Brigola/Heß, NuR 2017, 729, 732; Franzius, NuR 2019, 649, 653; Guckelberger, Deutsches Verwaltungsprozessrecht unter unionsrechtlichem Anpassungsdruck, 2017, S. 200.

108) OVG Hamburg, Beschl. v. 15.8.2018 - 1 Es 1/18.P, ZUR 2019, 37, 39; s. auch Berkemann, DVB1. 2020, 1, 11; Bunge, in: ders., UmwRG, 2. Aufl. 2019, §5 Rdnr. 27.

109) Ingold/Münkler, EurUP 2018, 468, 481.

110) OVG Hamburg, Beschl. v. 15.8.2018 - 1 Es 1/18.P, ZUR 2019, 37, 39; s. auch Schlacke, NVwZ 2017, 905, 910; dies., NVwZ 2019, 1392, 1395.

111) OVG Lüneburg, Urt. v. 31.7.2018 - 7 KS 17/16, Rdnr. 161; offengelassen auch von OVG Münster, Urt. v. 4.9.2017 - 11 D 14/14.AK, Rdnr. 161, juris; dazu, dass dies grundsätzlich in besonders gelagerten Konstellationen denkbar ist, Fellenberg/Schiller, in: Landmann/Rohmer, Umweltrecht, Stand: 6/2019, \5 UmwRG Rdnr. 20; eine subjektive Komponente bejahend Ingold/Münkler, EurUP 2018, 468, 481; Schlacke, NVwZ 2017, 905, 910; Storost, UPR 2018, 52, 56.

112) Bunge, in: ders., UmwRG, 2. Aufl. 2019, \$5 Rdnr. 21; Guckelberger, Deutsches Verwaltungsprozessrecht unter unionsrechtlichem Anpassungsdruck, 2017, S. $200 \mathrm{f}$.

113) OVG Münster, Beschl. v. 15.3.2018 - 8 B 736/17, Rdnr. 7 ff., juris.

114) OVG Hamburg, Beschl. v. 15.8.2018 - 1 Es 1/18.P, ZUR 2019, 37, 39.

115) OVG Greifswald, Beschl. v. 8.5.2018 - 3 M 22/16, Rdnr. 128, juris; s. auch Bunge, in: ders., UmwRG, 2. Aufl. 2019, \5 Rdnr. 8 und in Rdnr. 10 dazu, dass die Behörde die Vorschriften über die Beteiligung richtig angewendet haben muss.

116) S. auch Bunge, in: ders., UmwRG, 2. Aufl. 2019, \5 Rdnr. 3; Schlacke, NVwZ 2019, 1392, 1395; s. auch Storost, UPR 2018, $52,56$.

117) Guckelberger, Deutsches Verwaltungsprozessrecht unter unionsrechtlichem Anpassungsdruck, 2017, S. 201; Schlacke, NVwZ 2017, 905, 910; Siegel, NVwZ 2016, 337, 340; s. auch Storost, UPR 2018, 52, 56.

118) Durner, Rechtsgutachten zur Wiedereinführung der Präklusion v. 7.10.2019, Stand 16.12.2019, abrufbar unter https://www. netzwerk-bahnen.de/assets/files/news/2019/2019_10_07_durner_rechtsgutachten_wiedereinfuehrung-der-praeklusion.pdf, S. 45; Széchényi, BayVB1. 2016, 366, 368.

119) Guckelberger, Deutsches Verwaltungsprozessrecht unter unionsrechtlichem Anpassungsdruck, 2017, S. 200. 